\title{
LATTICES, EQUIVALENCE RELATIONS, AND SUBGROUPS
}

PHILIP M. WHITMAN

1. Introduction. A lattice ${ }^{1}$ is a set of elements $A, B, \cdots$ together with a relation $\leqq$ defined between some or all pairs of the elements such that $A \leqq A$ for each $A$; if $A \leqq B$ and $B \leqq A$ then $A=B$; if $A \leqq B$ and $B \leqq C$ then $A \leqq C$; and each pair of elements $A$ and $B$ have a least upper bound $A \cup B$ and a greatest lower bound $A \cap B$.

An equivalence relation ${ }^{2} R^{\prime}$ is a relation $\sim$ defined between some or all pairs of elements ("points") of a set $\subseteq$ such that $p \sim p$ for every point $p$; if $p \sim q$ then $q \sim p$; if $p \sim q$ and $q \sim r$ then $p \sim r$. If more than one relation is under discussion, we may specify the one used at the moment by writing " $p \sim q$ in $R^{\prime}$."

It is well known that the collection of all equivalence relations on a given set $\subseteq$ forms a lattice. Ore [4] has characterized lattices which are isomorphic to the lattice $\Omega^{*}$ of all equivalence relations on some set S. One could go farther and ask what lattices are isomorphic to sublattices of $\mathfrak{R}^{*}$. Our answer to this is: any lattice is isomorphic to a sublattice of the lattice of all equivalence relations on some set; more concisely, any lattice is a lattice of equivalence relations.

Garrett Birkhoff has shown [1] that any lattice of equivalence relations is isomorphic to a lattice of subgroups. Therefore the result stated in the previous paragraph implies: any lattice is isomorphic to a sublattice of the lattice of all subgroups of a suitable group.

2. Outline. The first and larger part of the paper relates lattices and equivalence relations. Since the formal construction and proof in this part are somewhat lengthy and complicated, we first outline the main steps and indicate the motivation.

A lattice $\mathfrak{R}$ is given; we wish to show that there is some set $\mathfrak{S}$, and some sublattice $\mathfrak{R}^{\prime}$ of the lattice $\mathfrak{R}^{*}$ of all equivalence relations on $\mathfrak{S}$, such that $\mathbb{R}$ and $\mathbb{R}^{\prime}$ are isomorphic.

We shall take $\mathfrak{S}$ as the union of disjoint subsets $\mathfrak{A}, \mathfrak{B}, \cdots$, one for each element $A, B, \ldots$ of $\mathfrak{\&}$. The equivalence relations $A^{\prime}, B^{\prime}, \ldots$ corresponding to $A, B, \cdots$ must be chosen in such a manner that

Presented to the Society, February 26, 1944 ; received by the editors April 25, 1944 and, in revised form, December 3, 1945.

${ }^{1}$ Sometimes called a structure. For a thorough discussion of lattices, see Birkhoff [2]. Numbers in brackets refer to the references cited at the end of the paper.

2 For a discussion of the relevant properties of equivalence relations (or congruence relations as they are sometimes called), see Birkhoff [1], Dubreil and Dubreil-Jacotin [3], or Ore [4]. 
$A \leqq B$ implies $A^{\prime} \leqq B^{\prime}$ and that $(A \cup B)^{\prime}=A^{\prime} \cup B^{\prime}$ and $(A \cap B)^{\prime}$ $=A^{\prime} \cap B^{\prime}$; this will make $\mathfrak{I}^{\prime}$ a homomorphic image of $\mathfrak{R}$. For an isomorphism, $A^{\prime} \leqq B^{\prime}$ must imply $A \leqq B$. To insure this we propose that the subset $\mathfrak{A}$ shall contain two points, $l(A)$ and $r(A)$, such that $l(A) \sim r(A)$ in $B^{\prime}$ if and only if $A \leqq B$. The notations $l(A)$ and $r(A)$ may be thought of as standing for "left end of $\mathfrak{Y}$ " and "right end of $\mathfrak{A}$." Similarly $\mathfrak{B}$ is to contain points $l(B)$ and $r(B)$ which are equivalent in $Z^{\prime}$ if and only if $B \leqq Z$, and so on. However, for simplicity we shall devote our attention to $\mathfrak{A}$ and the points and subsets thereof except when it becomes necessary to refer to $\mathfrak{B}$, and so on.

One's first thought might be to have $\mathfrak{A}$ consist merely of $l(A)$ and $r(A)$, and define $B^{\prime}$ insofar as it concerns points of $\mathfrak{A}$ by specifying $l(A) \sim r(A)$ in $B^{\prime}$ if and only if $A \leqq B$. But suppose $A \leqq C \cup D$ though neither $A \leqq C$ nor $A \leqq D$; by this definition one would have $l(A)$ and $r(A)$ equivalent in $(C \cup D)^{\prime}$ but not in $C^{\prime}$ or $D^{\prime}$ and so not in $C^{\prime} \cup D^{\prime}$, contradicting $C^{\prime} \cup D^{\prime}=(C \cup D)^{\prime}$. To overcome this difficulty we may enlarge $\mathfrak{A}$ to include another point $p(C, D)$ and specify that $l(A) \sim p(C, D)$ in $C^{\prime}$ and in $(C \cup D)^{\prime}$ while $p(C, D) \sim r(A)$ in $D^{\prime}$ and in $(C \cup D)^{\prime}$; we may think of $l(A)$ and $p(C, D)$ as being in this respect images of $l(C)$ and $r(C)$ and think of $p(C, D)$ and $r(A)$ as images of $l(D)$ and $r(D)$, with some images having been consolidated with each other or existing points for simplicity.

Now however we have introduced somewhat the same difficulty all over again (one step farther toward the background) for suppose $l(A) \sim p(C, D)$ in $C^{\prime}$ and $C \leqq E \cup F$. Then necessarily $C^{\prime} \leqq(E \cup F)^{\prime}$ so we need $l(A) \sim p(C, D)$ in $(E \cup F)^{\prime}=E^{\prime} \cup F^{\prime}$ yet we dare not (for instance) have this relation automatically hold in $E^{\prime}$, for otherwisesince $p(C, D) \sim r(A)$ in $D^{\prime}$-we shall get $l(A) \sim r(A)$ in $E^{\prime} \cup D^{\prime}=$ $(E \cup D)^{\prime}$ by transitivity and yet it need not be true that $A \leqq E \cup D$. So we must in a manner similar to that of the previous paragraph introduce a new point between $l(A)$ and $p(C, D)$. Evidently we can never stop this process at any finite point without having the same trouble; we are driven to making an infinite succession of enlargements of $\mathfrak{A}$ and hoping (or rather, proving) that their set union will have the desired properties though no one of them does.

In the above discussion we put our attention on one pair of elements $C$ and $D$ with $A \leqq C \cup D$. But of course if $A \leqq G \cup H$ then we must also introduce a point $p(G, H)$ in a similar manner. But now we have difficulty in trying to prove $(P \cap Q)^{\prime}=P^{\prime} \cap Q^{\prime}$. For it might be that

$$
p(C, D) \sim l(A) \sim p(G, H) \text { in } P^{\prime}
$$

while 


$$
p(C, D) \sim r(A) \sim p(G, H) \text { in } Q^{\prime} .
$$

Thus in $P^{\prime} \cap Q^{\prime}, p(C, D) \sim p(G, H)$ but these need not be equivalent to either $l(A)$ or $r(A)$, whereas in $(P \cap Q)^{\prime}$ by definition one or the other of the "paths" (1) or (2) must be followed. This trouble can be overcome by having not one but three images of $l(C), r(C), l(D)$, and so on. That is, instead of the one new point $p(C, D)$ we have $p^{i}(C, D), i=0,1,2,3,4,5,6$, where $p^{0}(C, D)=l(A)$ and $p^{6}(C, D)$ $=r(A)$ and $p^{i}(C, D) \sim p^{i+1}(C, D)$ in $C^{\prime}$ if $i$ is even and in $D^{\prime}$ if $i$ is odd. Also of course we must include additional points if $C \leqq E \cup F$, and so on, as discussed above. With this change, consider again the type of situation which was causing trouble; for instance

$$
\begin{aligned}
p^{3}(C, D) & \sim p^{2}(C, D) \sim p^{1}(C, D) \sim l(A) \\
& \sim p^{1}(G, H) \sim p^{2}(G, H) \text { in } P^{\prime}
\end{aligned}
$$

while

$$
\begin{aligned}
p^{3}(C, D) & \sim p^{4}(C, D) \sim p^{5}(C, D) \sim r(A) \sim p^{5}(G, H) \\
& \sim p^{4}(G, H) \sim p^{3}(G, H) \sim p^{2}(G, H) \text { in } Q^{\prime} .
\end{aligned}
$$

We propose to arrange matters so that $p^{i}(C, D) \sim p^{i+1}(C, D)$ in $J^{\prime}$ will imply $C \leqq J$ if $i$ is even and $D \leqq J$ if $i$ is odd. Then for instance $p^{3}(C, D) \sim p^{4}(C, D)$ in $Q^{\prime}$ will imply $p^{2}(C, D) \sim p^{1}(C, D)$ in $Q^{\prime}$ while $p^{4}(C, D) \sim p^{5}(C, D)$ in $Q^{\prime}$ will imply $p^{3}(C, D) \sim p^{2}(C, D)$ in $Q^{\prime}$ so that the possibility of the path (4) in $Q^{\prime}$ will imply that the equivalences (3) also hold in $Q^{\prime}$ and so also in $P^{\prime} \cap Q^{\prime}$ as desired.

We may describe the above construction in the terminology of electricity by saying that $l(A)$ and $r(A)$ are connected by several wires or paths in parallel, one path for each pair of lattice elements $C$ and $D$ with $A \leqq C \cup D$. Each path has six filters connected in series, three of which pass current (permit equivalence) of frequency (relation) $J^{\prime}$ if and only if $C \leqq J$ in the lattice, while the other three pass current in $J^{\prime}$ if and only if $D \leqq J^{\prime}$. The filters themselves are complex arrangements of a similar structure.

This gives a general idea of the procedure necessary; our task is now to make the construction precise and prove the existence of the properties that the construction is designed to provide.

3. Construction ${ }^{3}$ of the sets and equivalence relations. A lattice

3 If the given lattice is finite, Definitions 1-5 involve only finite processes; if the lattice is infinite, the word "construction" must be understood in a broader sense. But in both cases, Definitions 6-7 involve infinite processes. One hopes that a proof can be found which will involve only finite processes if the given lattice is finite. 
$\mathfrak{R}$ is given. We shall suppose that in addition to the lattice relation of inclusion, denoted $\leqq$, there is also a relation of preceding and following which linearly orders all elements of the lattice 4 (if the lattice has a finite number of elements, the existence of such a relation is trivial; otherwise we must invoke the axiom of choice). In Definitions 1-4 reference is made to a pair of elements $C$ and $D$ of the lattice; when writing the pair in these definitions we shall write first the one which "precedes" the other under the relation just discussed.

Definition 1. If $A \in \mathbb{R}$, denote by $\mathfrak{A}_{1}$ the set of points $p^{\mathbf{i}}(C, D)$ for $i=0,1,2,3,4,5,6$ and all pairs $C, D \in \mathbb{R}$ such that $A \leqq C \cup D$, where

$$
p^{0}(C, D)=p^{0}(G, H)=l(A)
$$

for all pairs $C, D$ and $G, H$ and likewise

$$
p^{\theta}(C, D)=p^{\theta}(G, H)=r(A)
$$

but otherwise $p^{i}(C, D) \neq p^{i}(G, H)$ if $i \neq j$ or if $C, D$ and $G, H$ are distinct pairs. Similarly, all points of $\mathfrak{A}_{1}$ are distinct from the points of $\mathfrak{B}_{1}, \mathfrak{E}_{1}, \cdots$.

Definition 2. The subset of $\mathfrak{A}_{1}$ consisting of the two points $p^{i}(C, D)$ and $p^{i+1}(C, D)$ is denoted $\left(C_{1}, D_{1}\right)^{i}$; the subset of $\mathfrak{A}_{1}$ consisting of $l(A)$ and $r(A)$ is denoted $A_{0} .\left(C_{0}, D_{0}\right)^{i}$ is a null set.

Obviously the subsets in Definition 2 overlap but this causes no trouble.

We now proceed to define by induction sets $\mathfrak{A}_{i}$. Suppose that for each $i(0 \leqq i<n)$ and each $A \in \mathfrak{R}$ there has been defined a set $\mathfrak{A}_{i}$ in such a manner that (for $1 \leqq i<n)$ if $A \leqq C \cup D$ then $\mathfrak{A}_{i}$ has subsets $\left(C_{i}, D_{i}\right)^{i}, j=0,1, \cdots, 5$, with $\left(C_{i-1}, D_{i-1}\right)^{i} \subset\left(C_{i}, D_{i}\right)^{i}$ and with the points of $\left(C_{i}, D_{i}\right)^{j}$ in one-to-one correspondence with those of $\mathfrak{C}_{i-1}$

"This relation of "preceding" and "following" is not important to the argument and is merely for convenience in stating Definitions 1-4.

b Strictly speaking, this notation should also include a symbol to indicate that the points are in the set corresponding to $A$, as distinguished from the sets corresponding to $B, C, \cdots$, but to simplify notation we shall fix our attention on the sets related to a fixed $A$ and subsets thereof, and on equivalence relations $A^{\prime}, B^{\prime}, \ldots$, only so far as they concern points of this set, unless otherwise indicated. We arrange that no point of the set corresponding to $A$ shall be equivalent to any point of the sets corresponding to $B, C, \cdots$. It is permitted that $C=D, D=A$, and so on. It would be possible to reduce the number of points in such cases, but the present definition will minimize the number of separate cases to be considered in proofs. The symbol $=$ when used between names of points means that the names stand for the same point; when used between names of lattice elements it means the names stand for the same element of the lattice. 
if $j$ is even and with those of $\mathfrak{D}_{i-1}$ if $j$ is odd, and with no overlapping except as required by these assumptions and Definition 2. Then for $n>1$, we have the following definition.

DEFINITION 3. $\mathfrak{A}_{n}$ consists of $\mathfrak{A}_{n-1}$ with each of the latter's subsets $\left(C_{n-1}, D_{n-1}\right)^{j}$ augmented by as many points as are in $\mathfrak{C}_{n-1}$ but not in $\mathfrak{S}_{n-2}$ (if $j$ is even) or in $\mathfrak{D}_{n-1}$ but not in $\mathfrak{D}_{n-2}$ (if $j$ is odd), the augmented subsets being called $\left(C_{n}, D_{n}\right)^{j}$.

It is to be observed that $\mathfrak{A}_{0}$ and $\mathfrak{P}_{1}$ satisfy the hypotheses, and that if the hypotheses hold then $\mathfrak{A}_{n}$ again has the same properties; thus the induction is effective. In view of the one-to-one correspondence specified, we may denote by $q^{-1}$ the point of $\mathfrak{E}_{n-1}$ or $\mathfrak{D}_{n-1}$ corresponding to $q \in\left(C_{n}, D_{n}\right)^{i}$. Because of the overlapping of the subsets of $\mathfrak{A}_{1}$ and hence of $\mathfrak{A}_{n},\left[p^{2}(C, D)\right]^{-1}$, for instance, may be taken as either $r(D)$ or $l(C)$ depending on whether we are at the moment concerned with $p^{2}(C, D)$ as a point of $\left(C_{n}, D_{n}\right)^{1}$ or as a point of $\left(C_{n}, D_{n}\right)^{2}$.

Corresponding to each $B \in R$, a relation $B_{1}{ }^{\prime}$ is next defined so far as it concerns points of $\mathfrak{A}$.

Definition 4. $p \sim q$ in $B_{1}{ }^{\prime}$ if there is some $A \in \mathbb{R}$ such that $p, q \in \mathfrak{P}_{1}$ and one of (5), (6) or (7) holds.

(5) For some pair $C, D \in \mathbb{Q}$ with $A \leqq C \cup D, p=p^{i}(C, D)$ and $q=p^{i+1}(C, D)$ or vice versa, and either

(5a) $i$ is even and $C \leqq B$ or

(5b) $i$ is odd and $D \leqq B$.

(6) $p=q$.

(7) There exist points $q_{1}, \cdots, q_{k}$ such that $q_{1}=p, q_{k}=q$, and for each $i(i=1,2, \cdots, k-1) q_{i}$ and $q_{i+1}$ satisfy the conditions of (5).

Suppose that, for $1 \leqq i<n$ and each $B \in \mathbb{R}$, equivalence relations $B_{i}{ }^{\prime}$ have been defined. Then by induction we define $B_{n}{ }^{\prime}$ :

Definition 5. $p \sim q$ in $B_{n}^{\prime}$ for $n>1$ if $p=q$ or there exist $A \in \mathbb{R}$ and points $q_{1}, q_{2}, \cdots, q_{k} \in \mathfrak{A}_{n}$ with $q_{1}=p, q_{k}=q$, and for each $i$ $(i=1, \cdots, k-1)$ there exist $j$ and $C, D \in \mathbb{R}$ with $A \leqq C \cup D ; q_{i}$, $q_{i+1} \in\left(C_{n}, D_{n}\right)^{j}$ and $q_{i}^{-1} \sim q_{i+1}^{-1}$ in $B_{n-1}{ }^{\prime}$.

It is to be observed that $B_{1}{ }^{\prime}$ is an equivalence relation, and that if $B_{n-1}{ }^{\prime}$ is an equivalence relation so is $B_{n}{ }^{\prime}$, so the induction is effective. Having thus defined relations with subscripts $1,2, \cdots$ and sets with subscripts $0,1,2, \cdots$ we now define ones without subscript, using $\sum$ to denote set union.

Definition 6. $\mathfrak{U}=\sum_{i=0}^{\infty} \mathfrak{A}_{i} ; \mathfrak{S}=\sum_{\mathrm{all} A} \mathfrak{A} ;(C, D)^{j}=\sum_{i=1}^{\infty}\left(C_{i}, D_{i}\right)^{j}$. 
Definition 7. $p \sim q$ in $B^{\prime}$ if for some $n, p \sim q$ in $B_{n}{ }^{\prime}$.

That $B^{\prime}$ as thus defined is an equivalence relation will appear shortly (Lemma 3).

Definition 8. $A$ point $s \in \mathfrak{A}_{1}$ is between points $p, q \in \mathfrak{A}$ if for some $C$ and $D, s \in(C, D)^{i}, p \in(C, D)^{j}$, and $q \in(C, D)^{k}$ with $j<i<k$ or $k<i<j$, or if $k=j+1$ and $s=p^{k}(C, D)$, or if $j=k+1$ and $s=p^{j}(C, D)$, provided in all cases that $p, q$, and $s$ are distinct.

For example, $p^{1}(C, D)$ is between $p^{2}(C, D)$ and $p^{0}(C, D)$, for we may take $i=1, j=2$, and $k=0$. Likewise $p^{5}(C, D)$ is between $p^{3}(C, D)$ and $r(A)$, for we may take $i=4, j=3$, and $k=5$ since $p^{5}(C, D)$ belongs to $(C, D)^{4}$ as well as to $(C, D)^{5}$. In fact, "between" means just what its name implies. One should emphasize the restriction in the definition that $s$ must be not only in $\mathfrak{A}$ but indeed in $\mathfrak{A}_{1}$; otherwise only a partial ordering would be possible.

4. Properties of $\mathbb{R}^{\prime}$. It is required that the equivalence relations $A^{\prime}, B^{\prime}, \cdots$ form a lattice $\mathfrak{R}^{\prime}$ isomorphic to the given lattice $\mathfrak{R}$. To prove that this requirement is satisfied, we must first examine in some detail the consequences of the above definitions, with attention to how it is possible to have $p \sim q$.

LEMma 1. If $A \leqq B$ then $l(A) \sim r(A)$ in $B_{1}{ }^{\prime}$.

Proof. Take $B=C=D$ in Definitions 1 and 4. Then in $B_{1}^{\prime}$, $l(A) \sim p^{1}(C, D) \sim p^{2}(C, D) \sim p^{3}(C, D) \sim p^{4}(C, D) \sim p^{5}(C, D) \sim r(A)$ by (5) so $l(A) \sim r(A)$ in $B_{1}^{\prime}$ by (7).

LemMa 2. If $p \sim q$ in $B_{m}{ }^{\prime}$ then $p \sim q$ in $B_{n}{ }^{\prime}$ for $m \leqq n$ and $p \sim q$ in $B^{\prime}$.

Proof. The case $m=n$ is trivial.

If $m=1$ but $n>1$, we proceed by induction on $n$; suppose the lemma is true for $n<\nu$ and we wish to prove it true for $n=\nu$. If $p \sim q$ in $B_{1}{ }^{\prime}$ then by Definition 4 there exist $A \in \mathbb{R}$ and $q_{1}, \cdots, q_{k} \in \mathfrak{Y}_{1}$ with $q_{1}=p$, $q_{k}=q$, and $q_{i} \sim q_{i+1}$ by (5), or else $p=q$. If $p=q$ they are equivalent in $B_{n}^{\prime}$ by Definition 5 , so suppose the other alternative holds. Now if $q_{i} \sim q_{i+1}$ by (5a) then $C \leqq B$; by Lemma $1, l(C) \sim r(C)$ in $B_{1}{ }^{\prime}$. By induction, $l(C) \sim r(C)$ in $B_{\nu-1}{ }^{\prime}$. By similar reasoning, if the equivalence in $B_{1}{ }^{\prime}$ is by $(5 \mathrm{~b})$ then $l(D) \sim r(D)$ in $B_{\nu-1}{ }^{\prime}$, so for all $i, q_{i}{ }^{-1} \sim q_{i+1}{ }^{-1}$ in $B_{\nu-1}{ }^{\prime}$; hence $p \sim q$ in $B_{\nu}{ }^{\prime}$ as desired, by Definition 5 .

If $m>1$, we proceed by induction on $m$; suppose $p \sim q$ in $B_{m}{ }^{\prime}$. By Definition 5 there exist $A \in \mathbb{R}$ and $q_{1}, \cdots, q_{k} \in \mathscr{P}_{m}$ with $q_{i}^{-1} \sim q_{i+1}^{-1}$ in $B_{m-1}{ }^{\prime}$ (as above, the case $p=q$ is trivial). But then by induction 
on $m, q_{i}^{-1} \sim q_{i+1}^{-1}$ in $B_{\nu-1}{ }^{\prime}$, for all $i$, so by Definition 5, $p \sim q$ in $B_{\nu}{ }^{\prime}$ as desired.

That the equivalence also holds in $B^{\prime}$ follows from Definition 7.

\section{LEMMA 3. $B^{\prime}$ is an equivalence relation.}

Proof. We must show that $B^{\prime}$ has the properties stated in $\$ 1$. Suppose first that $p=q$. Then $p$ and $q$ are in $\mathfrak{A}$ and hence in $\mathfrak{A}_{n}$ for some $A$ and $n$, by Definition 6 ; by Definition $5, p \sim q$ in $B_{n}{ }^{\prime}$ so, by Definition 7, $p \sim q$ in $B^{\prime}$ as desired. Suppose next that $p \sim q$ in $B^{\prime}$; then $p \sim q$ in $B_{n}{ }^{\prime}$ for some $n$, by Definition 7 ; since $B_{n}{ }^{\prime}$ is an equivalence relation, $q \sim p$ in $B_{n}{ }^{\prime}$ so $q \sim p$ in $B^{\prime}$, as desired. Suppose lastly that $p \sim q \sim r$ in $B^{\prime}$; then $p \sim q$ in $B_{n}{ }^{\prime}$ for some $n$ and $q \sim r$ in $B_{m}{ }^{\prime}$ for some $m$; by Lemma $2, p \sim q \sim r$ in $B_{\max (m, n)}{ }^{\prime}$ so $p \sim r$ in that relation which is known to be an equivalence relation; hence $p \sim r$ in $B^{\prime}$ as desired. Thus $B^{\prime}$ has all the properties required of an equivalence relation.

LEmma 4. If $p \sim q$ in $B_{n}{ }^{\prime}$ then there exist $A \in \mathbb{R}$ and $s_{1}, s_{2}, \cdots, s_{k} \in \mathfrak{A}_{n}$ such that $s_{1}=p, s_{k}=q, s_{i} \in \mathfrak{Y}_{1}(i \neq 1, k)$ and that the set of $s_{i}$ is minimal (that is, none can be omitted without violating the requirements placed on them) and that either $n=1$ and $s_{i} \sim s_{i+1}$ by (5) for all $i(i=1, \cdots$, $k-1)$ or else $n>1$ and $s_{i}$ and $s_{i+1}$ for all $i(i=1, \cdots, k-1)$ satisfy the requirements imposed on $q_{i}$ and $q_{i+1}$ in Definition 5.

Note. By Definitions 4 and 5 there exist points $q_{i}$ with these properties except $q_{2} \in \mathfrak{P}_{1}, i \neq 1, k$ (in those definitions a priori only $q_{i} \in \mathfrak{A}_{n}$ ), and minimality. Minimality. can easily be attained since the number of $q_{i}$ is finite, but it is more difficult to show that we can take $s_{i} \in \mathfrak{A}_{1}$, $i \neq 1, k$; that is, $s_{i}=p^{\nu}(C, D)$ for some $\nu, C$, and $D$ depending on $i$. It may also be remarked that by virtue of the requirement that $s_{i} \sim s_{i+1}$ by (5) or Definition 5-more precisely, by (5) or certain conditions stated in Definition 5-it follows that $s_{i}$ and $s_{i+1}$ must be in the same subset $(C, D)^{\nu}$ of $\mathfrak{A}$, for both in (5) and Definition 5 this is required. For $1<i \leqq k-2$, this fact and the requirement $s_{i} \in \mathfrak{A}_{1}, i \neq 1, k$, mean that $s_{i}=p^{\nu}(C, D)$ and either $s_{i+1}=p^{\nu+1}(C, D)$ or $s_{i+1}=p^{\nu-1}(C, D)$ for some $\nu$.

Proof. If $n=1$, the conclusion follows immediately from Definition 4 ; minimality can obviously be attained since only a finite number of points enter into (7).

Now proceed by induction on $n$; suppose $p \sim q$ in $B_{n}{ }^{\prime}$ and the lemma is known to be true for smaller values of the subscript. By Definition 5 there exist points $q_{i}=s_{i}$ with all the desired properties except minimality and the requirement $s_{i} \in \mathfrak{U}_{1}$ for $i \neq 1, k$. Suppose $q_{i} \notin \mathfrak{U}_{1}$ and $i \neq 1, k$; 
we shall show that $q_{i}$ can be omitted. But now in Definition $5, q_{i}$ and $q_{i+1}$ must be in the same subset of $\mathfrak{A}$, and $q_{i}^{-1} \sim q_{i+1}{ }^{-1}$ in $B_{n-1}{ }^{\prime}$ (again omitting the trivial case $p=q$ ); likewise $q_{i}$ and $q_{i-1}$ are in the same subset and $q_{i \rightarrow 1}{ }^{-1} \sim q_{i}^{-1}$ in $B_{n \rightarrow 1}{ }^{\prime}$. By Definitions 1, 2, and 3, $q_{i}$ can be in two subsets of $\mathfrak{A}$ only if $q_{i}=p^{j}(C, D)$ for some $j$, but then $q_{i}$ is in $\mathfrak{A}_{1}$ as desired. Otherwise, $q_{i}$ is in only one subset, and this one must also contain $q_{i \rightarrow 1}$ and $q_{i+1}$ and $q_{i \rightarrow 1}{ }^{-1} \sim q_{i}{ }^{-1} \sim q_{i+1}{ }^{-1}$ in $B_{n-1}{ }^{\prime}$. Hence $q_{i-1}{ }^{-1} \sim q_{i+1}{ }^{-1}$ in $B_{n-1}{ }^{\prime}$ so we may simply omit $q_{i}$ from the set of $q$ 's. Thus by omitting some of the points provided by Definition 5 we can obtain a set which satisfies the conclusions of the lemma.

Lemma 5. (i) If $p \in(C, D)^{i}$ and $q \in(C, D)^{j}$ and $p \sim q$ in $B_{n}{ }^{\prime}$ and $|i-j| \leqq 3$, then the points $s_{2}, \cdots, s_{k-1}$ of Lemma 4 may be taken as the set of all points of $\mathfrak{P}_{1}$ between $p$ and $q$.

(ii) If $p^{i}(C, D) \sim p^{i+1}(C, D)$ in $B_{n}{ }^{\prime}$ then $C \leqq B$ if $i$ is even, $D \leqq B$ if $i$ is odd.

(iii) If $l(A) \sim r(A)$ in $B_{n}{ }^{\prime}$ then $A \leqq B$.

Proof. First suppose $n=1$.

Suppose the hypotheses of (i) are satisfied. Since $n=1$, we may by Definitions 1 and 2 replace these hyptheses by the following which are implied by them : $p=p^{i}(C, D), q=p^{j}(C, D), p \sim q$ in $B_{1}{ }^{\prime}$, and $|i-i|$ $\leqq 4$; by "replace" we mean that one whole set of hypotheses replaces the other set, but $i$ and $j$ need not have the same meaning in both. Indeed, the fact that $(C, D)^{i}$ contains both $p^{i}(C, D)$ and $p^{i+1}(C, D)$ is the reason why in the second set we must allow a greater difference between $i$ and $j$. Since $n=1, \mathfrak{A}_{n}=\mathfrak{A}_{1}$, so in Lemma $4, s_{h} \in \mathfrak{A}_{1}$ for all $h$. As in previous lemmas, we may ignore the trivial case $p=q$.

First consider the case $p=p^{0}(C, D)$. Then by the assumptions, $q=p^{j}(C, D)$ with $1 \leqq j \leqq 4$. The $s_{h}$ of Lemma 4 are all in $\mathfrak{A}_{1}$ for $h \neq 1, k$. Moreover, $s_{h}$ and $s_{h+1}$ must be in the same subset of $\mathfrak{A}$; in particular $s_{k-1}$ is in the same subset as $s_{k}$, so $s_{k-1}$ is either $p^{j+1}(C, D)$ or $p^{j-1}(C, D)$. We shall assume the latter but the same type of proof would apply in the former case; likewise we shall assume $j$ odd but a similar argument would hold if $j$ were even. In Lemma $4, p^{j-1}(C, D) \sim p^{j}(C, D)$ in $B_{1}{ }^{\prime}$ implies by (5) that $C \leqq B$ since if $j$ is odd then $j-1$ is even. If $j-1=0$ then $p=s_{k-1}$ and (i) holds, the set referred to being vacuous; otherwise $s_{k-2}$ exists. Both $s_{k-2}=q$ and $s_{k-2}=s_{k-1}$ are prohibited by minimality, so by Lemma 4 and (5), $s_{k-2}=p^{j-2}(C, D)$ and $D \leqq B$. Thus both $D \leqq B$ and $C \leqq B$. Then by $(5), p^{\nu}(C, D) \sim p^{\nu+1}(C, D)$ in $B_{n}^{\prime}$ for all $\nu$ by (5), so that if we take $s_{2}, \cdots, s_{k-1}$ as the set of points of $\mathfrak{A}_{1}$ between $p$ and $q$, they will have all the properties required of them by Lemma 4 ; obviously they are minimal, since only successive 
$p^{\nu}(C, D)$ can be equivalent by (5). Thus we have disposed of the case $p=p^{0}(C, D)$.

Now consider the case $p=p^{1}(C, D)$. If $q=p^{0}(C, D)$, the previous paragraph applies in view of the symmetry of our definitions as far as $p$ and $q$ are concerned. Otherwise, $q=p^{i}(C, D)$ with $2 \leqq j \leqq 5$. Either $s_{k-1}=p^{j-1}(C, D)$ or $s_{k-1}=p^{j+1}(C, D)$. In the former case the argument of the previous paragraph is still applicable; in the latter case the argument fails if $j+1=6$. But then $D \leqq B$ by Lemma 4 and (5) since $p^{5}(C, D) \sim p^{6}(C, D)$; also, either $s_{2}=p^{0}(C, D)$ or $s_{2}=p^{2}(C, D)$. If $s_{2}=p^{0}(C, D)$ then by the same reasoning, $C \leqq B$; since we already have found $D \leqq B$, then as in the previous paragraph, $p^{\nu}(C, D) \sim p^{\nu+1}(C, D)$ for all $\nu$ and (i) holds. If on the other hand $s_{2}=p^{2}(C, D)$ then either $s_{2}=q$ and the desired result (i) holds immediately, or $s_{3}=p^{3}(C, D)$ by minimality and so by Lemma 4 and (5), $C \leqq B$ and then just as in the last sentence (i) holds. Thus the case $p=p^{1}(C, D)$ is disposed of.

If $p=p^{i}(C, D)$ for $i=5$ or $i=6$, the situation is symmetric to the cases $i=1$ and $i=0$ of the last two paragraphs. If $i=2,3,4$, the same argument as with $i=0$ may be used if $p$ and $q$ are interchanged, for the essential part of that argument was that $p$ was between $q$ and one end of $\mathfrak{A}$ and that $q$ was far enough from the other end.

Thus for $n=1$, (i) holds in all cases.

Now suppose the hypothesis of (ii) holds, with still $n=1$. By (i) which has been proved for this value of $n$, we then have $p^{i}(C, D)$ $\sim p^{i+1}(C, D)$ by (5); and (5) gives precisely the desired conclusion, proving (ii) for $n=1$.

Now suppose the hypothesis of (iii) holds, with still $n=1$. By Lemma $4, s_{1}=p, s_{2}=p^{1}(C, D)$ for some $C$ and $D$, and, by (5), $C \leqq B$. By minimality $s_{3}=p^{2}(C, D)$ so $D \leqq B$. Thus $A \leqq C \cup D \leqq B$ and (iii) holds.

For $n>1$ we proceed by induction; suppose the lemma has been proved for all smaller values of $n$, and that the hypotheses of (i) hold. For the moment let us suppose also that neither $p$ nor $q$ is in $\mathfrak{A}_{1}$, and consider the $s_{i}$ of Lemma 4 . Both $s_{2}$ and $s_{k-1}$ are in $\mathfrak{A}_{1}$. If we consider these two points in the role of $p$ and $q$, then the same argument as for $n=1$ may be applied, except that when there we appealed to (5), now we must look to Definition 5 as the way to have $s_{i} \sim s_{i+1}$ in $B_{n}{ }^{\prime}$.

Consider the case $s_{2}=p^{0}(C, D)$. Then $s_{k-1}=p^{i}(C, D)$, where just as with $n=1, j$ is not necessarily the $j$ of the statement of the lemma, but $1 \leqq j \leqq 4$. Then $s_{k-2}$ must be in the same subset of $\mathfrak{A}$ as $s_{k-1}$, so $s_{k-2}$ is either $p^{j+1}(C, D)$ or $p^{j-1}(C, D)$; we shall assume that it is the latter and that $j$ is odd. By Lemma 4 and Definition $5, p^{j-1}(C, D) \sim p^{j}(C, D)$ in $B_{n}{ }^{\prime}$ implies $l(C) \sim r(C)$ in $B_{n-1}{ }^{\prime}$. If $j-1=0$ then $s_{2}=s_{k-2}$ and (i) 
holds as applied to $s_{2}$ and $s_{k-1}$; otherwise $s_{k-3}$ exists and by minimality it is not $p$ so $s_{k \rightarrow 3}=p^{i-2}(C, D)$, and by the same argument as in the previous sentence, $l(D) \sim r(D)$ in $B_{n-1}{ }^{\prime}$. Hence if we take $s_{3}, \cdots, s_{k-2}$ as the set of points of $\mathfrak{A}_{1}$ between $s_{2}$ and $s_{k-1}$, they will have all the properties required for Lemma 4 , for $s_{h}{ }^{-1}$ and $s_{h+1}{ }^{-1}$ will be either $l(C)$ and $r(C)$ or $l(D)$ and $r(D)$ or vice versa, and these have been shown equivalent.

Now consider the case $s_{2}=p^{1}(C, D)$. If $s_{k-1}=p^{0}(C, D)$, the previous paragraph applies by symmetry. Otherwise $s_{k-1}=p^{i}(C, D)$ with $2 \leqq j \leqq 5$. Either $s_{k-2}=p^{j-1}(C, D)$ or $s_{k-2}=p^{j+1}(C, D)$. In the former case the argument of the previous paragraph is still applicable; in the latter case the argument fails if $j+1=6$. But then by Lemma 4 and Definition 5 we have $l(D) \sim r(D)$ in $B_{n-1}{ }^{\prime}$. Also, either $s_{3}=p^{0}(C, D)$ or $s_{3}=p^{2}(C, D)$. If $s_{3}=p^{0}(C, D)$ then by the same reasoning $l(C) \sim r(C)$ in $B_{n-1}{ }^{\prime}$; since we already found $l(D) \sim r(D)$, then as in the previous paragraph, (i) holds as applied to $s_{2}$ and $s_{k-1}$. If on the other hand $s_{3}=p^{2}(C, D)$ then either $s_{3}=s_{k-1}$ and the desired result holds immediately, or $s_{4}=p^{3}(C, D)$ by minimality and so from $s_{3} \sim s_{4}$ we have $l(C) \sim r(C)$ in $B_{n-1}{ }^{\prime}$, and just as in the last sentence (i) holds as applied to $s_{2}$ and $s_{k-1}$.

If $s_{2}=p^{i}(C, D)$ for $i>1$, the proof may be referred to the cases $i=0$ and $i=1$ above, just as was done for $n=1$. Thus (i) holds in all cases, as applied to $s_{2}$ and $s_{k-1}$; now we must show that (i) holds as applied to $p$ and $q$.

Since we assumed that neither $p$ nor $q$ was in $\mathfrak{A}_{1}, s_{2}$ must be in the same subset of $\mathfrak{A}$ as $p$ by the conditions of Lemma 4 , and $s_{k-1}$ in the same subset as $q$. Then by Definition $8, s_{3}, \cdots, s_{k-2}$ (which by the above may be taken between $s_{2}$ and $s_{k-1}$ ) will be between $p$ and $q$. Suppose however that $s_{2}$ is not between $p$ and $q$ though $s_{3}$ is. In view of the conditions imposed on the $s_{i}$ in Lemma $4, s_{2}$ and $s_{3}$ must then both be in the same subset of $\mathfrak{A}$ as $p ; p^{-1} \sim s_{2}{ }^{-1} \sim s_{3}{ }^{-1}$ so $p^{-1} \sim s_{3}{ }^{-1}$ and $s_{2}$ may be omitted. Likewise if $s_{k-1}$ is not between $p$ and $q$ it may be omitted. Thus (i) holds if neither $p$ nor $q$ is in $\mathfrak{A}_{1}$; the argument can readily be modified to take care of the possibility that one or both is in $\mathfrak{A}_{1}$.

Now suppose the hypothesis of (ii) holds. By (i), $p^{i}(C, D)$ $\sim p^{i+1}(C, D)$ in $B_{n}{ }^{\prime}$ implies that their antecedents are equivalent in $B_{n-1}{ }^{\prime}$; that is, $l(C) \sim r(C)$ or $l(D) \sim r(D)$ in $B_{n-1}{ }^{\prime}$ as $i$ is even or odd. But by (iii), which is assumed already proved for $B_{n-1}{ }^{\prime}$, this implies $C \leqq B$ or $D \leqq B$ as $i$ is even or odd, so (ii) holds.

Now suppose the hypothesis of (iii) holds. In Lemma $4, s_{1}=p=l(A)$; $s_{2}=p^{1}(C, D)$ for some $C$ and $D$ since $s_{2} \in \mathfrak{A}_{1}$ and $s_{2}$ must be in the same 
subset as $s_{1}$. But by (i), $l(A) \sim p^{1}(C, D)$ in $B_{n}{ }^{\prime}$ implies $C \leqq B$. Also $s_{3}=p^{2}(C, D)$ by minimality so similarly $D \leqq B$. Then $A \leqq C \cup D \leqq B$ so (iii) holds.

Lemma 6. Suppose $p \sim q$ in $B_{n}{ }^{\prime}, p \in(C, D)^{i} \subset \mathfrak{A}$, and $q \in(E, F)^{i} \subset \mathfrak{A}$, where $C, D$ and $E, F$ are distinct pairs.

(i) If $i>1$ then $p \sim r(A)$ in $B_{n}{ }^{\prime}$.

(ii) If $i<4$ then $p \sim l(A)$ in $B_{n}{ }^{\prime}$.

(iii) If $j>1$ then $q \sim r(A)$ in $B_{n}{ }^{\prime}$.

(iv) If $j<4$ then $q \sim l(A)$ in $B_{n}{ }^{\prime}$.

Proof. Suppose the hypothesis of (i) holds: $i>1$. By Lemma 4 there exist points $s_{h}$ with the properties specified there; in particular, $s_{h} \in \mathfrak{A}_{1}$ for $h \neq 1, k$. We shall suppose that $p$ is not in $\mathfrak{A}_{1}$; the necessary modifications if $p \in \mathfrak{A}_{1}$ will be obvious. By Definition 2, either $s_{2}=p^{i}(C, D)$ or $s_{2}=p^{i+1}(C, D)$; we shall first suppose the latter. If $s_{2}=r(A)$, that is, $i=5$, then the conclusion of (i) holds immediately; otherwise by minimality $s_{3}=p^{i+2}(C, D)$. If $s_{3}=r(A)$ then (i) holds; otherwise $s_{4}=p^{i+3}(C, D)$. But $p^{i+1}(C, D) \sim p^{i+2}(C, D) \sim p^{i+3}(C, D)$ in $B_{n}{ }^{\prime}$ imply $C \leqq B$ and $D \leqq B$ by Lemma 5 (ii), so $p^{\nu}(C, D) \sim p^{p+1}(C, D)$ for all $\nu$, and $s_{2} \sim r(A)$ in $B_{n}{ }^{\prime}$. Since $p \sim s_{2}$ we have $p \sim r(A)$ as desired.

If $s_{2}=p^{i}(C, D)$ the same type of argument applies; the hypothesis $i>1$ insures that we shall get both $C \leqq B$ and $D \leqq B$.

Thus (i) holds. The proofs of (ii), (iii), and (iv) are similar.

5. Proof of isomorphism. Having developed some tools in the previous section, we now proceed to prove that the partially ordered system of equivalence relations of Definition 7 is a lattice (a sublattice of the lattice of all equivalence relations on (5) and is isomorphic to the given lattice $\&$. Using the natural ordering among equivalence relations, we write $B^{\prime} \leqq G^{\prime}$ if and only if $p \sim q$ in $B^{\prime}$ implies $p \sim q$ in $G^{\prime}$.

Lemma 7. If $B \leqq G$ then $B^{\prime} \leqq G^{\prime}$.

Proof. From Definition 4 it is obvious that $B \leqq G$ implies $B_{1}{ }^{\prime} \leqq G_{1}{ }^{\prime}$. By Definition 5 and induction it follows that then $B_{n}{ }^{\prime} \leqq G_{n}{ }^{\prime}$, so by Definition $7, B^{\prime} \leqq G^{\prime}$.

Thus Lemma 7 holds; this proves that there is an order-homomorphism between the lattices. To prove it a lattice-homomorphism we need the next two lemmas.

Any two equivalence relations, $G^{\prime}$ and $H^{\prime}$, have ${ }^{6}$ a greatest lower bound, $G^{\prime} \cap H^{\prime}$, as equivalence relations-namely, $p \sim q$ in $G^{\prime} \cap H^{\prime}$ if and only if $p \sim q$ in both $G^{\prime}$ and $H^{\prime}$-though it is not apparent a priori

- See for instance Birkhoff [1], or the other references cited in footnote 2. 
that this new equivalence relation is one of those which we have constructed to correspond to the elements of the given lattice. Likewise $G^{\prime}$ and $H^{\prime}$ have a least upper bound $G^{\prime} \cup H^{\prime}-p \sim q$ in $G^{\prime} \cup H^{\prime}$ if and only if there exist $r_{1}, r_{2}, \cdots, r_{k}$ with $r_{1}=p, r_{k}=q$, and for each $i$, $r_{i} \sim r_{i+1}$ in $G^{\prime}$ or in $H^{\prime}$-which need not a priori correspond to an element of the given lattice, but we proceed to show that it is in fact identical with the relation constructed to correspond to $G \cup H$.

Lemma 8. $(G \cup H)^{\prime}=G^{\prime} \cup H^{\prime}$.

Proof. $G \leqq G \cup H$ so, by Lemma $7, G^{\prime} \leqq(G \cup H)^{\prime}$; similarly $H^{\prime}$ $\leqq(G \cup H)^{\prime}$; hence $G^{\prime} \cup H^{\prime} \leqq(G \cup H)^{\prime}$ since $G^{\prime} \cup H^{\prime}$ is the least upper bound of $G^{\prime}$ and $H^{\prime}$ as equivalence relations. As for the converse, we propose to prove by induction on $n$ that

$$
(G \cup H)_{n}{ }^{\prime} \leqq G_{n+1}{ }^{\prime} \cup H_{n+1}{ }^{\prime} .
$$

Suppose $n=1$. If $p \sim q$ in $(G \cup H)_{1}{ }^{\prime}$ then by Lemma 4 there exist the usual points $s_{j}$ with $s_{j} \sim s_{j+1}$ by (5). Suppose it is the alternative (5a) which holds; then for an even $i, s_{j}=p^{i}(C, D), s_{j+1}=p^{i+1}(C, D)$ or vice versa, and $C \leqq G \cup H$. Now consider the set $\mathscr{C}_{1}$; it has subsets $(G, H)^{\nu}$ since $C \leqq G \cup H$, and $p^{\nu}(G, H) \sim p^{\nu+1}(G, H)$ in $G_{1}{ }^{\prime}$ if $\nu$ is even and in $H_{1}{ }^{\prime}$ if $\nu$ is odd, by Definition 4. By Definition 5 the images of these points in $\mathfrak{A}_{2}$ are equivalent in $G_{2}{ }^{\prime}$ or $H_{2}{ }^{\prime}$ as the case may be; if the prefix $I$ denotes their images, then

$$
\begin{aligned}
s_{j} & =p^{i}(C, D)=I p^{0}(G, H) \sim I p^{1}(G, H) \sim \cdots \sim I p^{5}(G, H) \sim I p^{6}(G, H) \\
& =p^{i+1}(C, D)=s_{j+1}
\end{aligned}
$$

in $G_{2}{ }^{\prime} \cup H_{2}{ }^{\prime}$. Thus for each $j, s_{j} \sim s_{j+1}$ in $G_{2}{ }^{\prime} \cup H_{2}{ }^{\prime}$ so $p \sim q$ in $G_{2}{ }^{\prime} \cup H_{2}{ }^{\prime}$ as desired.

If $n>1$, we proceed by induction. If $p \sim q$ in $(G \cup H)_{n}{ }^{\prime}$ then by Lemma 4 there exist the usual $s_{j}$ with $s_{j}{ }^{-1} \sim s_{j+1}{ }^{-1}$ in $(G \cup H)_{n-1}{ }^{\prime}$ by Definition 5. By induction, $s_{j}{ }^{-1} \sim s_{j+1}{ }^{-1}$ in $G_{n}{ }^{\prime} \cup H_{n}{ }^{\prime}$. By the nature of the least upper bound, there exist $r_{1}, \cdots, r_{k}$ in $\mathbb{S}$ or in $\mathfrak{D}$ with $r_{1}=s_{j}^{-1}, r_{k}=s_{j+1^{-1}}, r_{i} \sim r_{i+1}$ in $G_{n}{ }^{\prime}$ or in $H_{n}{ }^{\prime}$. By Definition 5, their images in $\mathfrak{A}$ are equivalent: $I r_{i} \sim I r_{i+1}$ in $G_{n+1}{ }^{\prime}$ or in $H_{n+1}{ }^{\prime}$. Hence

$$
s_{j}=I r_{1} \sim I r_{k}=s_{j+1} \text { in } G_{n+1}{ }^{\prime} \cup H_{n+1}{ }^{\prime},
$$

for each $j$. Hence $p \sim q$ in $G_{n+1}{ }^{\prime} \cup H_{n+1}{ }^{\prime}$, as desired.

Thus for all $n,(G \cup H)_{n}{ }^{\prime} \leqq G_{n+1}{ }^{\prime} \cup H_{n+1}{ }^{\prime}$. But $G_{n+1}{ }^{\prime} \leqq G^{\prime}$ and $H_{n+1}{ }^{\prime} \leqq H^{\prime}$ so

$$
(G \cup H)_{n}{ }^{\prime} \leqq G_{n+1}{ }^{\prime} \cup H_{n+1}{ }^{\prime} \leqq G^{\prime} \cup H^{\prime} .
$$

If $p \sim q$ in $(G \cup H)^{\prime}$ then $p \sim q$ in $(G \cup H)_{n}{ }^{\prime}$ for some $n$ by Definition 7, 
and then by the above inclusion relations, $p \sim q$ in $G^{\prime} \cup H^{\prime}$. This proves Lemma 8.

LEMma 9. $(G \cap H)^{\prime}=G^{\prime} \cap H^{\prime}$.

Proof. By Lemma 7, $(G \cap H)^{\prime} \leqq G^{\prime}$ and $(G \cap H)^{\prime} \leqq H^{\prime}$, so $(G \cap H)^{\prime}$ $\leqq G^{\prime} \cap H^{\prime}$. For the converse, suppose $p \sim q$ in $G^{\prime} \cap H^{\prime}$. Then $p \sim q$ in $G^{\prime}$ and in $H^{\prime}$. By Definition $7, p \sim q$ in $G_{n}{ }^{\prime}$ and in $H_{m}{ }^{\prime}$ for some $n$ and $m$; suppose for instance $n \geqq m$; then $p \sim q$ in $G_{n}{ }^{\prime}$ and in $H_{n}{ }^{\prime}$. It will therefore prove the lemma if we can show that $p \sim q$ in $G_{n}{ }^{\prime}$ and in $H_{n}{ }^{\prime}$ imply $p \sim q$ in $(G \cap H)_{n}{ }^{\prime}$, which we proceed to do by induction.

Suppose $n=1$.

Case 1. $p=p^{i}(C, D), q=p^{j}(C, D),|i-j| \leqq 3$. Now $p \sim q$ in $G_{1}{ }^{\prime}$ and in $H_{1}{ }^{\prime}$. By Lemma 5 (i), the $s_{h}$ of Lemma 4 for both $G_{1}{ }^{\prime}$ and $H_{1}{ }^{\prime}$ may be taken as the set of points of $\mathfrak{U}_{1}$ between $p$ and $q$, and $s_{h} \sim s_{h+1}$ by (5a) or (5b), depending on whether the index is even or odd. Hence $C \leqq G$ and $C \leqq H$, or $D \leqq G$ and $D \leqq H$-depending on whether it is (5a) or (5b) that applies-so $C \leqq G \cap H$ or $D \leqq G \cap H$. Hence by (5), $s_{h} \sim s_{h+1}$ in $(G \cap H)_{1}{ }^{\prime}$. This holds for each $h$, so $p \sim q$ in $(G \cap H)_{1}{ }^{\prime}$ as desired.

Case 2. $p=p^{i}(C, D), q=p^{j}(C, D),|i-j|>3$. We may assume $i<j$. By Lemma 4 there exist the usual $s_{h}$ with $s_{h} \sim s_{h+1}$ in $G_{n}^{\prime}$ by (5). Then $i=0$ or $s_{2}=p^{i-1}(C, D)$ or $s_{2}=p^{i+1}(C, D)$.

Case 2a. $s_{2}=p^{i+1}(C, D)$. Suppose for instance $i$ is even; then by (5), $C \leqq G$. Also $s_{3}=s_{1}$ and $s_{3}=s_{2}$ are excluded by minimality, and $s_{2}=q$ and $s_{3}=q$ are excluded by $|i-j|>3$, so $s_{3}=p^{i+2}(C, D)$ and $D \leqq G$ by (5). Hence using (5) in the opposite direction, $p^{\nu}(C, D) \sim p^{\nu+1}(C, D)$ for all $\nu$; and $p, l(A), r(A)$, and $q$ are all equivalent in $G_{n}{ }^{\prime}$. Before proceeding, we bring the other subcases of Case 2 to a corresponding situation.

Case 2b. $s_{2}=p^{i-1}(C, D)$. If $i=1$, we have immediately $p^{0}(C, D)$ $\sim p^{1}(C, D)$ and $C \leqq G$. Otherwise $i=2$, since $i>2$ is excluded by $|i-j|>3$. But by minimality,

$$
s_{3}=p^{i-2}(C, D)=l(A)
$$

and $C \leqq G$ and $D \leqq G$ from $s_{2} \sim s_{3}$ and $s_{1} \sim s_{2}$.

Case 2c. $i=0$. Here $p=l(A)$.

Thus in all subcases of Case 2 , if $p^{\nu}(C, D)$ and $p^{\nu+1}(C, D)$ are both between $p$ and $l(A)$ then $C \leqq G$ or $D \leqq G$ as $\nu$ is even or odd. Similarly $C \leqq H$ or $D \leqq H$, so $C \leqq G \cap H$ or $D \leqq G \cap H$, and $p^{\nu}(C, D) \sim p^{\nu+1}(C, D)$ in $(G \cap H)_{1}{ }^{\prime}$, so $p \sim l(A)$ in $(G \cap H)_{1}{ }^{\prime}$, as well as in $G_{1}{ }^{\prime}$ and $H_{1}{ }^{\prime}$. Similarly $q \sim r(A)$ in $G_{1}{ }^{\prime}$, in $H_{1}{ }^{\prime}$, and in $(G \cap H)_{1}{ }^{\prime}$. Then 


$$
l(A) \sim p \sim q \sim r(A) \text { in } G_{1}^{\prime} \text { and in } H_{1}^{\prime} ;
$$

by Lemma 5 (iii), $A \leqq G$ and $A \leqq H$. Hence $A \leqq G \cap H$, so by Lemma 1 , $l(A) \sim r(A)$ in $(G \cap H)_{1}^{\prime}$. Thus

$$
p \sim l(A) \sim r(A) \sim q \text { in }(G \cap H)_{1}^{\prime}
$$

as desired.

Case 3. $p \in(C, D)^{i}, q \in(E, F)^{j}$ with $C, D$ and $E, F$ distinct pairs. This breaks up into four overlapping subcases.

Case 3a. $i>1, j>1$. By Lemma $6, p \sim r(A) \sim q$ in $G_{1}{ }^{\prime}$ and in $H_{1}{ }^{\prime}$. By Cases 1 and 2, $p \sim r(A) \sim q$ in $(G \cap H)_{1}{ }^{\prime}$ so $p \sim q$ in $(G \cap H)_{1}{ }^{\prime}$ as desired.

Case 3b. $i>1, j<4$. By Lemma 6, $p \sim r(A)$ and $q \sim l(A)$ in $G_{1}{ }^{\prime}$ and in $H_{1}{ }^{\prime}$; hence $r(A) \sim l(A)$ in $G_{1}{ }^{\prime}$ and in $H_{1}{ }^{\prime}$. By Lemma 5 (iii), $A \leqq G$ and $A \leqq H$ so $A \leqq G \cap H$ and by Lemma $1, l(A) \sim r(A)$ in $(G \cap H)_{1}{ }^{\prime}$. By Cases 1 and $2, p \sim r(A)$ and $q \sim l(A)$ in $(G \cap H)_{1}{ }^{\prime}$, so by transitivity $p \sim q$ in $(G \cap H)_{1}{ }^{\prime}$ as desired.

Cases $3 \mathrm{c}$ and $3 \mathrm{~d}$. $i<4, j>1$ or $j<4$. Similar to Cases $3 \mathrm{a}$ and $3 \mathrm{~b}$.

This concludes the proof for $n=1$. If $n>1$, we proceed by induction.

Case 1. $p \in(C, D)^{i}, q \in(C, D)^{j},|i-j| \leqq 3$. By Lemma 5 (i), the $s_{h}$ of Lemma 4 for both $G_{n}{ }^{\prime}$ and $H_{n}{ }^{\prime}$ may be taken as the points of $\mathfrak{A}_{1}$ between $p$ and $q$. But $s_{h}{ }^{-1} \sim s_{h+1}{ }^{-1}$ in $G_{n-1}{ }^{\prime}$ and in $H_{n-1}{ }^{\prime}$ and (by induction) in $(G \cap H)_{n-1}{ }^{\prime}$, for all $h$. By Definition $5, p \sim q$ in $(G \cap H)_{n}{ }^{\prime}$.

Case 2. $p \in(C, D)^{i}, q \in(C, D)^{j},|i-j|>3$. We may suppose $i<j$. Let us also suppose that neither $p$ nor $q$ is in $\mathfrak{A}_{1}$; the modifications otherwise necessary will be apparent. By Lemma 4 the usual $s_{h}$ exist, with $s_{h}{ }^{-1} \sim s_{h+1}{ }^{-1}$ in $G_{n-1}{ }^{\prime}$, and $s_{2} \in(C, D)^{i}$ but $s_{3} \notin(C, D)^{i}$ by minimality. Also $s_{h} \in \mathfrak{A}_{1}$ for $h \neq 1, k$. Then $s_{3} \in(C, D)^{i+1}$ or $s_{3} \in(C, D)^{i-1}$ or $i=0$.

Case 2a. $s_{3} \in(C, D)^{i+1}$. Then $s_{2}=p^{i}(C, D)$ and $s_{3}=p^{i+1}(C, D)$ is impossible, for this would put $s_{1}, s_{2}$, and $s_{3}$ in the same $(C, D)^{i}$ contrary to minimality. Hence, $s_{2}=p^{i+1}(C, D)$ and $s_{3}=p^{i+2}(C, D)$. If, say, $i$ is even, then $s_{2}{ }^{-1} \sim s_{3}{ }^{-1}$ implies $l(D) \sim r(D)$ in $G_{n-1}{ }^{\prime}$. Likewise $s_{4}=p^{i+3}(C, D)$ and $l(C) \sim r(C)$ in $G_{n-1}{ }^{\prime}$. Also $p^{-1} \sim s_{2}{ }^{-1}$ and by transitivity $p^{-1} \sim l(C)$ in $G_{n-1}{ }^{\prime}$; if $i$ were odd this last would be $p^{-1} \sim l(D)$ in $G_{n \rightarrow 1}{ }^{\prime}$.

Case 2b. $s_{3} \in(C, D)^{i-1}$. In a similar manner, if $p^{\nu}(C, D)$ and $p^{\nu+1}(C, D)$ are between $p$ and $l(A)$ then $l(C) \sim r(C)$ or $l(D) \sim r(D)$ in $G_{n-1}{ }^{\prime}$, as $\nu$ is even or odd, and $p^{-1} \sim l(C)$ or $p^{-1} \sim l(D)$ in $G_{n-1}{ }^{\prime}$.

Case 2c. $i=0$. In a similar manner, the same conclusion holds as in Case $2 b$.

Thus in all subcases of Case 2 , if $p^{\nu}(C, D)$ and $p^{\nu+1}(C, D)$ are between $p$ and $l(A)$ then $l(C) \sim r(C)$ or $l(D) \sim r(D)$ in $G_{n-1}^{\prime}$ as $\nu$ is even 
or odd, and $p^{-1} \sim l(C)$ or $p^{-1} \sim l(D)$ in $G_{n-1}{ }^{\prime}$ as $i$ is even or odd. Similarly these equivalences hold in $H_{n-1}{ }^{\prime}$ and so by induction in $(G \cap H)_{n-1}{ }^{\prime}$; hence $p \sim l(A)$ in $G_{n}{ }^{\prime}$, in $H_{n}{ }^{\prime}$, and in $(G \cap H)_{n}{ }^{\prime}$. Similarly $q \sim r(A)$ in $G_{n}{ }^{\prime}$, in $H_{n}{ }^{\prime}$, and in $(G \cap H)_{n}{ }^{\prime}$. Then

$$
l(A) \sim p \sim q \sim r(A) \text { in } G_{n}^{\prime} \text { and in } H_{n}^{\prime} ;
$$

by Lemma 5 (iii), $A \leqq G$ and $A \leqq H$ so $A \leqq G \cap H$ and by Lemmas 1 and $2, l(A) \sim r(A)$ in $(G \cap H)_{n}{ }^{\prime}$. Thus

$$
p \sim l(A) \sim r(A) \sim q \text { in }(G \cap H)_{n}^{\prime}
$$

as desired.

Case 3. $p \in(C, D)^{i}, q \in(E, F)^{i}$. The same argument as for $n=1$ may be used.

Thus Lemma 9 is proved. This shows that we have a lattice-homomorphism. To prove this an isomorphism we need the following lemma.

LEMMA 10. If $B^{\prime} \leqq G^{\prime}$ then $B \leqq G$.

Proof. By Lemma $1, l(B) \sim r(B)$ in $B^{\prime}$, so by hypothesis $l(B) \sim r(B)$ in $G^{\prime}$. By Definition $7, l(B) \sim r(B)$ in $G_{n}{ }^{\prime}$ for some $n$. By Lemma 5 (iii), $B \leqq G$ as desired.

From Lemmas 7-10, the equivalence relations which were constructed form a lattice isomorphic to the given lattice. Hence we have the main result:

THEOREM 1. Any lattice is isomorphic to a sublattice of the lattice of all equivalence relations on some set.

CoROllary 1. If a lattice identity holds in the lattice of all equivalence relations on the set $\mathfrak{\Im}$, for every $\mathfrak{\Im}$, then this identity holds in every lattice.

6. Connection with groups. Birkhoff has shown [1] that any sublattice of the lattice of all equivalence relations on a set is isomorphic to a sublattice of the lattice of all subgroups of some group. ${ }^{7}$ In view of Theorem 1, we immediately have the following theorem.

THEOREM 2. Any lattice is isomorphic to a sublattice of the lattice of all subgroups of some group.

CoROLlaRY 2. If a lattice identity holds in the lattice of all subgroups of the group (\$), for every $\$$, then this identity holds in every lattice.

7 The converse is better known; Birkhoff [1] has a proof of it as well as references. 
Since Birkhoff gives only an indication of the proof of his result, it is perhaps worth giving a proof here. We are given a lattice of equivalence relations on a set $\mathfrak{S}$. If $B$ is one of these relations, let $B^{*}$ be the group of all permutations $P: p \rightarrow f(p)$ of the points $p$ of $\subseteq$ such that

(8) for each $p, f(p) \sim p$ in $B$, and

(9) $P$ leaves unchanged all but a finite number of the points of $\mathfrak{\Im}$.

Evidently $B^{*}$ is a subgroup of the group of all permutations of $\mathfrak{S}$.

Then $B^{*} \cap C^{*}=(B \cap C)^{*}$, for any permutation which satisfies (9) and has $f(p) \sim p$ in $B$ and in $C$ also has $f(p) \sim p$ in $B \cap C$ and conversely.

Also $B^{*} \cup C^{*}=(B \cup C)^{*}$. For certainly $B^{*} \leqq(B \cup C)^{*}$ and $C^{*} \leqq(B \cup C)^{*}$; on the other hand suppose $P \in(B \cup C)^{*}$; that is, $P$ is a permutation which involves only a finite number of points and in which $f(p) \sim p$ in $B \cup C$. By definition of join of equivalence relations, there exist for each $p$ affected a finite number of points $w_{1}, \cdots, w_{k}$ with $w_{1}=p, w_{k}=f(p)$, and $w_{i} \sim w_{i+1}$ in $B$ or in $C$. Consider, for a given $p$, the permutation which is the following product:

$$
\left(w_{1} w_{2}\right)\left(w_{2} w_{3}\right)\left(w_{3} w_{4}\right) \cdots\left(w_{k-1} w_{k}\right)\left(w_{k-2} w_{k-1}\right)\left(w_{k-3} w_{k-2}\right) \cdots\left(w_{1} w_{2}\right) .
$$

We observe that this product equals $\left(w_{1} w_{k}\right)$; that is, it simply transposes $p$ and $f(p)$. On the other hand each factor $\left(w_{i} w_{i+1}\right)$ is a member of $B^{*}$ or $C^{*}$ according as $w_{i} \sim w_{i+1}$ in $B$ or in $C$. Thus any transposition $\left(w_{1} w_{k}\right)$ of points equivalent under $B \cup C$ is a member of $B^{*} \cup C^{*}$. But by (9), any member of $(B \cup C)^{*}$ affects only a finite number of points of $\mathfrak{S}$, and hence is the product of a finite number of transpositions $(p q)$; it is evident that these transpositions can be so chosen that $p \sim q$ in $B \cup C$; by the above argument each of these transpositions (and so also their product) is in $B^{*} \cup C^{*}$, so $(B \cup C)^{*} \leqq B^{*} \cup C^{*}$. Then by the first part of this paragraph, $(B \cup C)^{*}=B^{*} \cup C^{*}$. Thus the given lattice of equivalence relations has an isomorphic lattice of subgroups as asserted.

\section{REFERENCES}

1. Garrett Birkhoff, On the structure of abstract algebras, Proc. Cambridge Philos. Soc. vol. 31 (1935) pp. 433-454.

2. - Lattice theory, Amer. Math. Soc. Colloquium Publications, vol. 25, New York, 1940.

3. Paul Dubreil and Marie-Louise Dubreil-Jacotin, Thếorie algébrique des relations d'équivalence, Journal de Mathématiques (9) vol. 18 (1939) pp. 63-95.

4. Oystein Ore, Theory of equivalence relations, Duke Math. J. vol. 9 (1942) pp. 573-627.

The University of Pennsylvania 